UDC 517.5

V. Ryazanov (Inst. Appl. Math. and Mech. Nat. Acad. Sci. Ukraine, Donetsk),

U. Srebro (Technion — Israel Inst. Technol., Haifa, Israel),

E. Yakubov (Holon Acad. Inst. Technol., Israel)

\title{
ON THE THEORY OF THE BELTRAMI EQUATION
}

\section{ДО ТЕОРІЇ РІВНЯННЯ БЕЛЬТРАМІ}

We study ring homeomorphisms and, on this basis, obtain a series of theorems on existence of the so-called ring solutions for degenerate Beltrami equations. A general statement on the existence of solutions for the Beltrami equations is formulated extending earlier results. In particular, we give new existence criteria of homeomorphic solutions $f$ of the class $W_{\text {loc }}^{1,1}$ with $f^{-1} \in W_{\text {loc }}^{1,2}$ in terms of tangential dilatations and functions of finite mean oscillation. The ring solutions also satisfy additional capacity inequalities.

Вивчаються кільцеві гомеоморфізми, і на цій підставі отримано низку теорем про існування так званих кільцевих розв'язків вироджених рівнянь Бельтрамі. Сформульовано загальне твердження про існування розв' язків рівнянь Бельтрамі, що узагальнює більш ранні результати. Зокрема, наведено нові критерії існування гомеоморфних розв' язків $f$ класу $W_{\text {loc }}^{1,1} f^{-1} \in W_{\text {loc }}^{1,2}$ у термінах тангенціальних дилатацій та функцій скінченного середнього коливання. Кільцеві розв' язки задовольняють також додаткові ємнісні нерівності.

1. Introduction. As known, the Beltrami equation plays an important role in the mapping theory. The main goal of this paper is to present general principles which allow to obtain variety of conditions for the existence of homeomorphic ACL solutions in the degenerate case. Our existence theorems are proved by an approximation method.

Let $D$ be a domain in the complex plane $\mathbb{C}$, i.e., open and connected subset of $\mathbb{C}$, and let $\mu: D \rightarrow \mathbb{C}$ be a measurable function with $|\mu(z)|<1$ a.e. The Beltrami equation is the equation of the form

$$
f_{\bar{z}}=\mu(z) \cdot f_{z}
$$

where $f_{\bar{z}}=\bar{\partial} f=\left(f_{x}+i f_{y}\right) / 2, f_{z}=\partial f=\left(f_{x}-i f_{y}\right) / 2, z=x+i y$, and $f_{x}$ and $f_{y}$ are partial derivatives of $f$ in $x$ and $y$, correspondingly. The function $\mu$ is called the complex coefficient and

$$
K_{\mu}(z)=\frac{1+|\mu(z)|}{1-|\mu(z)|}
$$

the maximal dilatation or in short the dilatation of the equation (1). The Beltrami equation (1) is said to be degenerate if

$$
\operatorname{ess} \sup K_{\mu}(z)=\infty \text {. }
$$

An ACL homeomorphism $f: D \rightarrow \mathbb{C}$ is called a ring solution of the Beltrami equation (1) if $f$ satisfies (1) a.e., $f^{-1} \in W_{\text {loc }}^{1,2}$ and $f$ is a ring $Q$-homeomorphism at every point $z_{0} \in D$ with $Q(z)=K_{\mu}^{T}\left(z, z_{0}\right)$ where the function

$$
K_{\mu}^{T}\left(z, z_{0}\right)=\frac{\left|1-\frac{\overline{z-z_{0}}}{z-z_{0}} \mu(z)\right|^{2}}{1-|\mu(z)|^{2}}
$$

is called the tangential dilatation with respect to $z_{0}$, cf. [1-3]. For the definition of ring $Q$-homeomorphisms, see Section 2. The terms have been first introduced in [4].

Recall that a mapping $f: D \rightarrow \mathbb{C}$ is absolutely continuous on lines, abbr. $f \in$ ACL, if, for every closed rectangle $R$ in $D$ whose sides are parallel to the coordinate axes, $f \mid R$ is absolutely continuous on almost all line segments in $R$ which are parallel to the sides of 
$R$. In particular, $f$ is ACL if it belongs to the Sobolev class $W_{\text {loc }}^{1,1}$, see e.g. [5, p. 8]. Note that, if $f \in \mathrm{ACL}$, then $f$ has partial derivatives $f_{x}$ and $f_{y}$ a.e. and, thus, by the well-known Gehring - Lehto theorem every ACL homeomorphism $f: D \rightarrow \mathbb{C}$ is totally differentiable a.e., see [6] or [7, p. 128]. For a sense-preserving ACL homeomorphism $f: D \rightarrow \mathbb{C}$, the Jacobian $J_{f}(z)=\left|f_{z}\right|^{2}-\left|f_{\bar{z}}\right|^{2}$ is nonnegative a.e., see [7, p. 10]. In this case, the complex dilatation $\mu_{f}$ of $f$ is the ratio $\mu(z)=f_{\bar{z}} / f_{z}$, if $f_{z} \neq 0$ and $\mu(z)=0$ otherwise, and the dilatation $K_{f}$ of $f$ is $K_{\mu}(z)$, see (2), and, thus, $|\mu(z)| \leq 1$ and $K_{\mu}(z) \geq 1$ a.e.

Note that every homeomorphic ACL solution $f$ of the Beltrami equation with $K_{\mu} \in$ $\in L_{\text {loc }}^{1}$ belongs to the class $W_{\text {loc }}^{1,1}$ as in all our theorems. Indeed,

$$
\left|f_{z}\right|+\left|f_{\bar{z}}\right|=K_{\mu}^{1 / 2}(z) J_{f}^{1 / 2}(z)
$$

and by Hölder's inequality, on every compact set $C \subset D$,

$$
\left\|f_{\bar{z}}\right\|_{1} \leq\left\|f_{z}\right\|_{1} \leq\left\|K_{\mu}\right\|_{1}^{1 / 2} A(f(C))^{1 / 2}
$$

where $A(f(C))$ is the area of the set $f(C)$. Hence $f \in W_{\text {loc }}^{1,1}$, see e.g. [5, p. 8]. Similarly, it is easy to show that, if $K_{\mu} \in L_{\mathrm{loc}}^{p}, p \in[1, \infty]$, then $f \in W_{\mathrm{loc}}^{1, s}$ where $s=2 p /(1+p) \in$ $\in[1,2]$.

Note also that the condition $f^{-1} \in W_{\text {loc }}^{1,2}$ given in the definition of a ring solution implies that a.e. point $z$ is a regular point for the mapping $f$, i.e., $f$ is differentiable at $z$ and $J_{f}(z) \neq 0$, see Remark 2. Finally, note that the condition $K_{\mu} \in L_{\text {loc }}^{1}$ is necessary for a homeomorphic ACL solution $f$ of (1) to have the property $g=f^{-1} \in W_{\text {loc }}^{1,2}$ because this property implies that

$$
\int_{C} K_{\mu}(z) d x d y \leq 4 \int_{C} \frac{d x d y}{1-|\mu(z)|^{2}}=4 \int_{f(C)}\left|g_{w}\right|^{2} d u d v<\infty
$$

for every compact set $C \subset D$.

Recall that a real valued function $\varphi \in L_{\text {loc }}^{1}(D)$ is said to be of bounded mean oscillation in $D$, abbr. $\varphi \in \mathrm{BMO}(D)$ or simply $\varphi \in \mathrm{BMO}$, if

$$
\|\varphi\|_{*}=\sup _{B \subset D} f_{B}\left|\varphi(z)-\varphi_{B}\right| d x d y<\infty
$$

where the supremum is taken over all disks $B$ in $D$ and

$$
\varphi_{B}=\int_{B} \varphi(z) d x d y=\frac{1}{|B|} \int_{B} \varphi(z) d x d y
$$

is the mean value of the function $\varphi$ over $B$. It is well-known that $L^{\infty}(D) \subset \operatorname{BMO}(D) \subset$ $\subset L_{\text {loc }}^{p}(D)$ for all $1 \leq p<\infty$, see e.g. [8]. A function $\varphi$ in BMO is said to have vanishing mean oscillation, abbr. $\varphi \in \mathrm{VMO}$, if the above supremum taken over all disks $B$ in $D$ with $|B|<\varepsilon$ converges to 0 as $\varepsilon \rightarrow 0$.

The BMO space was introduced by John and Nirenberg, see [9], and soon became one of the main concepts in harmonic analysis, complex analysis and partial differential equations. BMO functions are related in many ways to quasiconformal and quasiregular mappings, see e.g. [10-15], and to mappings with finite distortion, see e.g. [16-19]. VMO has been introduced by Sarason, see [20]. A large number of papers are devoted to 
the study of existence, uniqueness and properties of solutions for various kind of differential equations and, in particular, of elliptic type with VMO coefficients, see e.g. [21 - 24]. In this connection, it should be noted that by a recent result of Brezis and Nirenberg in [25] the Sobolev class $W_{\text {loc }}^{1,2}$ is a subclass of VMO, see also [26].

Let $D$ be a domain in the complex plane $\mathbb{C}$. We say that a function $\varphi: D \rightarrow \mathbb{R}$ has finite mean oscillation at a point $z_{0} \in D$, abbr. $f$ of FMO at $z_{0}$, if

$$
d_{\varphi}\left(z_{0}\right)=\varlimsup_{\varepsilon \rightarrow 0} f_{D\left(z_{0}, \varepsilon\right)}\left|\varphi(z)-\bar{\varphi}_{\varepsilon}\left(z_{0}\right)\right| d x d y<\infty
$$

where

$$
\bar{\varphi}_{\varepsilon}\left(z_{0}\right)=f_{D\left(z_{0}, \varepsilon\right)} \varphi(z) d x d y<\infty
$$

is the mean value of the function $\varphi(z)$ over the disk $D\left(z_{0}, \varepsilon\right)$ with small $\varepsilon>0$. Thus, the notion includes the assumption that $\varphi$ is integrable in some neighborhood of the point $z_{0}$. We call $d_{\varphi}\left(z_{0}\right)$ the dispersion of the function $\varphi$ at the point $z_{0}$. We say that a function $\varphi: D \rightarrow \mathbb{R}$ is of finite mean oscillation in $D$, abbr. $\varphi \in \operatorname{FMO}(D)$ or simply $\varphi \in \mathrm{FMO}$, if $\varphi$ has a finite dispersion at every point $z \in D$. We call also the number

$$
d_{\varphi}\left(z_{0}, \varepsilon_{0}\right)=\sup _{\varepsilon \in\left(0, \varepsilon_{0}\right]} f_{D\left(z_{0}, \varepsilon\right)}\left|\varphi(z)-\bar{\varphi}_{\varepsilon}\left(z_{0}\right)\right| d x d y<\infty
$$

the maximal dispersion of the function $\varphi$ in the disk $D\left(z_{0}, \varepsilon_{0}\right)$.

Below we use the notations $D(r)=D(0, r)=\{z \in \mathbb{C}:|z|<r\}$ and

$$
A\left(\varepsilon, \varepsilon_{0}\right)=\left\{z \in \mathbb{C}: \varepsilon<|z|<\varepsilon_{0}\right\} .
$$

Lemma 1. Let $D \subset \mathbb{C}$ be a domain such that $D\left(e^{-1}\right) \subset D$, and let $\varphi: D \rightarrow \mathbb{R}$ be a nonnegative function. If $\varphi$ is integrable in $D\left(e^{-1}\right)$ and of FMO at 0 , then

$$
\int_{A\left(\varepsilon, e^{-1}\right)} \frac{\varphi(z) d x d y}{\left(|z| \log \frac{1}{|z|}\right)^{2}} \leq C \log \log \frac{1}{\varepsilon}
$$

for $\varepsilon \in\left(0, e^{-e}\right)$ where

$$
C=2 \pi\left[2 \varphi_{0}+3 e^{2} d_{0}\right]
$$

$\varphi_{0}$ is the mean value of $\varphi$ over the disk $D\left(e^{-1}\right)$ and $d_{0}$ is the maximal dispersion of $\varphi$ in $D\left(e^{-1}\right)$.

Versions of this lemma have been first established for BMO functions in [27, 28] and then for FMO functions in [29] and [30, p. 251] (Lemma 2.15).

Conditions for the existence and uniqueness of ACL homeomorphic solutions for the Beltrami equation can be given in terms of the maximal dilatation $K_{\mu}(z)$. It was shown for instance that if $K_{\mu}(z)$ has a BMO or FMO majorant, then the Beltrami equation (1) has a homeomorphic ACL solution, see e.g. [27, 28, 30, 31], cf. [32, 33]. Various conditions for the existence of solutions for the Beltrami equation have been formulated in terms of integral and measure constraints on $K_{\mu}$, see e.g. [32-39]. These conditions assume either exponential integrability or at least high local integrability of the dilatation 
$K_{\mu}$. Here, as in [2-4], the existence criteria are expressed in terms of the tangential dilatations $K_{\mu}^{T}\left(z, z_{0}\right)$.

In [30], we proved that if $K_{\mu}(z)$ has a majorant $Q(z)$ of finite mean oscillation in $D$, then (1) has a homeomorphic ACL solution. Now we prove a stronger result, namely, the existence of a ring solution of (1) where the assumption on an FMO majorant for $K_{\mu}$ in $D$ is replaced by the weaker condition that every point $z_{0} \in D$ has a neighborhood $U_{z_{0}}$ and a function $Q_{z_{0}}: U_{z_{0}} \rightarrow[0, \infty]$ which is of finite mean oscillation at $z_{0}$ such that $K_{\mu}^{T}\left(z, z_{0}\right) \leq Q_{z_{0}}(z)$ for all $z \in U_{z_{0}}$, see Theorem 4 in Section 3 below. This as well as other new existence theorems established here are based on a general existence principle, Lemma 3. Some of these existence theorems are expressed in terms of the mean and the logarithmic mean of the tangential dilatation $K_{\mu}^{T}\left(z, z_{0}\right)$ over infinitesimal disks and annuli centered at $z_{0}$, see e.g. Theorems 5 and 6 . We also use Lemma 3 in a new proof of an extension of Lehto's theorem, Theorem 7, that we have first established in [4]. Finally, note that a series of interesting distortion theorems have been formulated in various terms in Section 2, see Remark 3 in the end of the work.

2. Distortion estimates for ring $Q$-homeomorphisms. Below we use the standard conventions $a / \infty=0$ for $a \neq \infty$ and $a / 0=\infty$ if $a>0$ and $0 \cdot \infty=0$, see e.g. [40, p. 6].

Given a measurable function $Q: D \rightarrow[1, \infty]$, we say that a homeomorphism $f: D \rightarrow$ $\rightarrow \overline{\mathbb{C}}$ is a $Q$-homeomorphism if

$$
M(f \Gamma) \leq \int_{D} Q(z) \cdot \rho^{2}(z) d x d y
$$

holds for every path family $\Gamma$ in $D$ and each $\rho \in \operatorname{adm} \Gamma$. This term was introduced in $[41-$ 43].

Recall that, given a family of paths $\Gamma$ in $\overline{\mathbb{C}}$, a Borel function $\rho: \overline{\mathbb{C}} \rightarrow[0, \infty]$ is called admissible for $\Gamma$, abbr. $\rho \in \operatorname{adm} \Gamma$, if

$$
\int_{\gamma} \rho(z)|d z| \geq 1
$$

for each $\gamma \in \Gamma$. The modulus of $\Gamma$ is defined by

$$
M(\Gamma)=\inf _{\rho \in \operatorname{adm} \Gamma} \int_{\mathbb{C}} \rho^{2}(z) d x d y .
$$

We say that a property $P$ holds for almost every (a.e.) path $\gamma$ in a family $\Gamma$ if the subfamily of all paths in $\Gamma$ for which $P$ fails has modulus zero. In particular, almost all paths in $\mathbb{C}$ are rectifiable.

Given a domain $D$ and two sets $E$ and $F$ in $\overline{\mathbb{C}}, \Gamma(E, F, D)$ denotes the family of all paths $\gamma:[a, b] \rightarrow \overline{\mathbb{C}}$ which join $E$ and $F$ in $D$, i.e., $\gamma(a) \in E, \gamma(b) \in F$ and $\gamma(t) \in D$ for $a<t<b$. We set $\Gamma(E, F)=\Gamma(E, F, \overline{\mathbb{C}})$ if $D=\overline{\mathbb{C}}$. A ring domain, or shortly a ring in $\overline{\mathbb{C}}$ is a doubly connected domain $R$ in $\overline{\mathbb{C}}$. Let $R$ be a ring in $\overline{\mathbb{C}}$. If $C_{1}$ and $C_{2}$ are the connected components of $\overline{\mathbb{C}} \backslash R$, we write $R=R\left(C_{1}, C_{2}\right)$. The capacity of $R$ can be defined by

$$
\operatorname{cap} R\left(C_{1}, C_{2}\right)=M\left(\Gamma\left(C_{1}, C_{2}, R\right)\right) \text {, }
$$

see e.g. [44]. Note that 


$$
M\left(\Gamma\left(C_{1}, C_{2}, R\right)\right)=M\left(\Gamma\left(C_{1}, C_{2}\right)\right),
$$

see e.g. Theorem 11.3 in [45].

Motivated by the ring definition of quasiconformality in [46], we introduce the following notion that localizes and extends the notion of a $Q$-homeomorphism. Let $D$ be a domain in $\mathbb{C}, z_{0} \in D, r_{0} \leq \operatorname{dist}\left(z_{0}, \partial D\right)$ and $Q: D\left(z_{0}, r_{0}\right) \rightarrow[0, \infty]$ a measurable function in the disk

$$
D\left(z_{0}, r_{0}\right)=\left\{z \in \mathbb{C}:\left|z-z_{0}\right|<r_{0}\right\}
$$

Set

$$
\begin{gathered}
A\left(r_{1}, r_{2}, z_{0}\right)=\left\{z \in \mathbb{C}: r_{1}<\left|z-z_{0}\right|<r_{2}\right\}, \\
C\left(z_{0}, r_{i}\right)=\left\{z \in \mathbb{C}:\left|z-z_{0}\right|=r_{i}\right\}, \quad i=1,2 .
\end{gathered}
$$

We say that a homeomorphism $f: D \rightarrow \overline{\mathbb{C}}$ is a ring Q-homeomorphism at the point $z_{0}$ if

$$
\operatorname{cap} R\left(f C_{1}, f C_{2}\right) \leq \int_{A} Q(z) \cdot \eta^{2}\left(\left|z-z_{0}\right|\right) d x d y
$$

for every annulus $A=A\left(r_{1}, r_{2}, z_{0}\right), 0<r_{1}<r_{2}<r_{0}$, and for every measurable function $\eta:\left(r_{1}, r_{2}\right) \rightarrow[0, \infty]$ such that

$$
\int_{r_{1}}^{r_{2}} \eta(r) d r=1 .
$$

Note that every $Q$-homeomorphism $f: D \rightarrow \overline{\mathbb{C}}$ is a ring $Q$-homeomorphism at each point $z_{0} \in D$. The next proposition gives other conditions on $f$ which force it to be a ring $Q$-homeomorphism, see Theorem 2.17 in [4].

Proposition 1. Let $f: D \rightarrow \mathbb{C}$ be a sense-preserving homeomorphism of the class $W_{\mathrm{loc}}^{1,2}$ such that $f^{-1} \in W_{\mathrm{loc}}^{1,2}$. Then at every point $z_{0} \in D$ the mapping $f$ is a ring $Q$ homeomorphism with $Q(z)=K_{\mu}^{T}\left(z, z_{0}\right)$ where $\mu(z)=\mu_{f}(z)$.

If $f$ is a plane $W_{\text {loc }}^{1,2}$ homeomorphism with a locally integrable $K_{f}(z)$, then $f^{-1} \in$ $\in W_{\text {loc }}^{1,2}$, see e.g. [47]. Hence we obtain the following consequence of Proposition 1.

Corollary 1. Let $f: D \rightarrow \mathbb{C}$ be a sense-preserving homeomorphism of the class $W_{\mathrm{loc}}^{1,2}$ and suppose that $K_{f}(z)$ is integrable in a disk $D\left(z_{0}, r_{0}\right) \subset D$ for some $z_{0} \in D$ and $r_{0}>0$. Then $f$ is a ring $Q$-homeomorphism at the point $z_{0} \in D$ with $Q(z)=K_{\mu}^{T}\left(z, z_{0}\right)$ where $\mu(z)=\mu_{f}(z)$.

Note also the convergence theorem which plays an important role in our scheme for deriving the existence theorems of the Beltrami equation, see Theorem 2.22 in [4].

Proposition 2. Let $f_{n}: D \rightarrow \overline{\mathbb{C}}, n=1,2, \ldots$, be a sequence of ring $Q$-homeomorphisms at a point $z_{0} \in D$. If $f_{n}$ converge locally uniformly to a homeomorphism $f: D \rightarrow$ $\rightarrow \overline{\mathbb{C}}$, then $f$ is also a ring $Q$-homeomorphism at the point $z_{0}$.

For points $z, \zeta \in \overline{\mathbb{C}}$, the spherical (chordal) distance $s(z, \zeta)$ between $z$ and $\zeta$ is given by

$$
s(z, \zeta)=\frac{|z-\zeta|}{\left(1+|z|^{2}\right)^{\frac{1}{2}}\left(1+|\zeta|^{2}\right)^{\frac{1}{2}}} \quad \text { if } \quad z \neq \infty \neq \zeta,
$$

ISSN 1027-3190. Укр. мат. журн., 2006, m.58, № 11 


$$
s(z, \infty)=\frac{1}{\left(1+|z|^{2}\right)^{\frac{1}{2}}} \quad \text { if } \quad z \neq \infty .
$$

Given a set $E \subset \mathbb{C}, \delta(E)$ denotes the spherical diameter of $E$, i.e.,

$$
\delta(E)=\sup _{z_{1}, z_{2} \in E} s\left(z_{1}, z_{2}\right) .
$$

Given a number $\Delta \in(0,1)$, a domain $D \subset \mathbb{C}$, a point $z_{0} \in D$, a number $r_{0} \leq$ $\leq \operatorname{dist}\left(z_{0}, \partial D\right)$, and a measurable function $Q: D\left(z_{0}, r_{0}\right) \rightarrow[0, \infty]$, let $\mathfrak{R}_{Q}^{\Delta}$ denote the class of all ring $Q$-homeomorphisms $f: D \rightarrow \overline{\mathbb{C}}$ at $z_{0}$ such that

$$
\delta(\overline{\mathbb{C}} \backslash f(D)) \geq \Delta .
$$

Next, we introduce the classes $\mathfrak{B}_{Q}^{\Delta}$ and $\mathfrak{F}_{Q}^{\Delta}$ of certain qc mappings. Let $\mathfrak{B}_{Q}^{\Delta}$ denote the class of all quasiconformal mappings $f: D \rightarrow \overline{\mathbb{C}}$ satisfying (16) such that

$$
K_{\mu}^{T}\left(z, z_{0}\right)=\frac{\left|1-\frac{\overline{z-z_{0}}}{z-z_{0}} \mu(z)\right|^{2}}{1-|\mu(z)|^{2}} \leq Q(z) \text { a.e. in } D\left(z_{0}, r_{0}\right)
$$

where $\mu=\mu_{f}$. Similarly, let $\mathfrak{F}_{Q}^{\Delta}$ denote the class of all quasiconformal mappings $f: D \rightarrow$ $\rightarrow \overline{\mathbb{C}}$ satisfying (16) such that

$$
K_{\mu}(z)=\frac{1+|\mu(z)|}{1-|\mu(z)|} \leq Q(z) \quad \text { a.e. in } \quad D\left(z_{0}, r_{0}\right) .
$$

Remark 1. Note that

$$
\mathfrak{F}_{Q}^{\Delta} \subset \mathfrak{B}_{Q}^{\Delta} \subset \mathfrak{R}_{Q}^{\Delta}
$$

Lemma 2. Let $f \in \mathfrak{R}_{Q}^{\Delta}$ and $\psi_{\varepsilon}:(0, \infty) \rightarrow[0, \infty], 0<\varepsilon<r_{0}$, a one parameter family of measurable functions such that

$$
0<I(\varepsilon)=\int_{\varepsilon}^{r_{0}} \psi_{\varepsilon}(t) d t<\infty, \quad \varepsilon \in\left(0, r_{0}\right) .
$$

Then, for all $\zeta \in D\left(z_{0}, r_{0}\right)$,

$$
s\left(f(\zeta), f\left(z_{0}\right)\right) \leq \frac{32}{\Delta} \exp \left(-\frac{2 \pi}{\omega\left(\left|\zeta-z_{0}\right|\right)}\right)
$$

where

$$
\omega(\varepsilon)=\frac{1}{I^{2}(\varepsilon)} \int_{A(\varepsilon)} Q(z) \psi_{\varepsilon}^{2}\left(\left|z-z_{0}\right|\right) d x d y
$$

and $A(\varepsilon)=A\left(\varepsilon, r_{0}, z_{0}\right)=\left\{z \in \mathbb{C}: \varepsilon<\left|z-z_{0}\right|<r_{0}\right\}$.

Proof. Set $C=\left\{z \in \mathbb{C}:\left|z-z_{0}\right|=\left|\zeta-z_{0}\right|\right\}$ and $C_{0}=\left\{z \in \mathbb{C}:\left|z-z_{0}\right|=r_{0}\right\}$. Then

$$
s\left(f(\zeta), f\left(z_{0}\right)\right) \leq \frac{32}{\Delta} \exp \left(-\frac{2 \pi}{\operatorname{cap} R\left(f C, f C_{0}\right)}\right)
$$

for every $f \in \mathfrak{R}_{Q}^{\Delta}$ by Lemma 3.21 in [4]. Thus, choosing $\eta(r)=\psi_{\varepsilon}(r) / I(\varepsilon), r \in\left(\varepsilon, r_{0}\right)$, in (14), we come to (21). 
Theorem 1. Let $f \in \mathfrak{R}_{Q}^{\Delta}$ and $\psi:[0, \infty] \rightarrow[0, \infty]$ a measurable function such that

$$
0<\int_{\varepsilon}^{r_{0}} \psi(t) d t<\infty, \quad \varepsilon \in\left(0, r_{0}\right) .
$$

Suppose that

$$
\int_{\varepsilon<\left|z-z_{0}\right|<r_{0}} Q(z) \psi^{2}\left(\left|z-z_{0}\right|\right) d x d y \leq C \int_{\varepsilon}^{r_{0}} \psi(t) d t
$$

for all $\varepsilon \in\left(0, r_{0}\right)$. Then

$$
s\left(f(\zeta), f\left(z_{0}\right)\right) \leq \frac{32}{\Delta} \exp \left(-\frac{2 \pi}{C} \int_{\left|\zeta-z_{0}\right|}^{r_{0}} \psi(t) d t\right)
$$

whenever $\zeta \in D\left(z_{0}, r_{0}\right)$.

In particular, Theorem 1 which is an immediate consequence of Lemma 2 makes possible to estimate the distortion in terms of the mean value of $Q$ over balls and its maximal dispersion, see (7).

Theorem 2. Let $f \in \mathfrak{R}_{Q}^{\Delta}$. Then

$$
s\left(f(\zeta), f\left(z_{0}\right)\right) \leq \frac{32}{\Delta}\left(\log \frac{e \varepsilon_{0}}{\left|\zeta-z_{0}\right|}\right)^{-\frac{1}{\beta_{0}}}
$$

for every point $\zeta \in D\left(z_{0}, e^{1-e} \varepsilon_{0}\right)$ where

$$
\beta_{0}=2 q_{0}+3 e^{2} d_{0},
$$

$q_{0}$ is the mean value and $d_{0}$ the maximal dispersion of $Q(z)$ in $D\left(z_{0}, \varepsilon_{0}\right)$.

Proof. With no loss of generality we may assume that $Q$ has finite mean oscillation at $z_{0}$ and integrable over a disk $D\left(z_{0}, \varepsilon_{0}\right)$ because otherwise (26) is trivial. The mean value and the dispersion of a function over disks are invariant under linear transformations $w=\left(z-z_{0}\right) / e \varepsilon_{0}$. Hence, (26) follows by Lemma 1 and Theorem 1 with $\psi(t)=1 /(t \log 1 / t)$.

Choosing in Lemma 2 a special functional parameter $\psi(t)$ we obtain also the following distortion theorem for ring $Q$-homeomorphisms in terms of the mean value of $Q$ over spheres.

Theorem 3. Let $f \in \mathfrak{R}_{Q}^{\Delta}$. Then

$$
s\left(f(\zeta), f\left(z_{0}\right)\right) \leq \frac{32}{\Delta} \exp \left(-\int_{\left|\zeta-z_{0}\right|}^{r_{0}} \frac{d r}{r q(r)}\right)
$$

for all $\zeta \in D\left(z_{0}, r_{0}\right)$ where $q(r)$ is the mean of $Q(z)$ over the circle $\left|z-z_{0}\right|=r$.

Proof. With no loss of generality we may assume that the integral $I \neq 0$ in (28) because otherwise (28) is trivial and that $I \neq \infty$ because otherwise we can replace $Q(z)$ by $Q(z)+\delta$ with arbitrarily small $\delta>0$ and then take the limit as $\delta \rightarrow 0$ in (28). Note that $\mathfrak{R}_{Q}^{\Delta} \subset \mathfrak{R}_{Q+\delta}^{\Delta}$. The condition $I \neq \infty$ implies, in particular, that $q(r) \neq 0$ a.e. in $\left(\varepsilon, r_{0}\right), \varepsilon=\left|\zeta-z_{0}\right|$. Set 


$$
\psi(t)= \begin{cases}1 /[t q(t)], & t \in\left(\varepsilon, r_{0}\right) \\ 0, & \text { otherwise }\end{cases}
$$

Then

$$
\int_{A} Q(z) \psi^{2}\left(\left|z-z_{0}\right|\right) d x d y=2 \pi I
$$

where $A=A\left(\varepsilon, r_{0}, z_{0}\right)=\left\{z \in \mathbb{C}: \varepsilon<\left|z-z_{0}\right|<r_{0}\right\}$.

Another consequence of Lemma 2 can be formulated in terms of the logarithmic mean of $Q$ over an annulus $A(\varepsilon)=A\left(\varepsilon, \varepsilon_{0}, z_{0}\right)=\left\{z \in \mathbb{C}: \varepsilon<\left|z-z_{0}\right|<\varepsilon_{0}\right\}$ which is defined by

$$
M_{\log }^{Q}(\varepsilon)=f_{\varepsilon}^{\varepsilon_{0}} q(t) d \log t:=\frac{1}{\log \varepsilon_{0} / \varepsilon} \int_{\varepsilon}^{\varepsilon_{0}} q(t) \frac{d t}{t}
$$

where $q(t)$ denotes the mean value of $Q$ over the circle $\left|z-z_{0}\right|=t$. Choosing in the expression (21) $\psi_{\varepsilon}(t)=1 / t$ for $0<\varepsilon<\varepsilon_{0}$, and setting $\varepsilon=\left|\zeta-z_{0}\right|$ we have the following statement.

Corollary 2. Let $Q: D\left(z_{0}, r_{0}\right) \rightarrow[0, \infty], r_{0} \leq \operatorname{dist}\left(z_{0}, \partial D\right)$, be a measurable function, $\varepsilon_{0} \in\left(0, r_{0}\right)$ and $\Delta>0$. If $f \in \mathfrak{R}_{Q}^{\Delta}$, then

$$
s\left(f(\zeta), f\left(z_{0}\right)\right) \leq \frac{32}{\Delta}\left(\frac{\left|\zeta-z_{0}\right|}{\varepsilon_{0}}\right)^{1 / M_{l o g}^{Q}\left(\left|\zeta-z_{0}\right|\right)}
$$

for all $\zeta \in D\left(z_{0}, \varepsilon_{0}\right)$.

\section{A general existence lemma and corollaries.}

Lemma 3. Let $\mu: D \rightarrow \mathbb{C}$ be a measurable function with $|\mu(z)|<1$ a.e. and $K_{\mu} \in L_{\mathrm{loc}}^{1}$. Suppose that for every $z_{0} \in D$ there exist $\varepsilon_{0} \leq \operatorname{dist}\left(z_{0}, \partial D\right)$ and a family of measurable functions $\psi_{z_{0}, \varepsilon}:(0, \infty) \rightarrow(0, \infty), \varepsilon \in\left(0, \varepsilon_{0}\right)$, such that

$$
0<I_{z_{0}}(\varepsilon):=\int_{\varepsilon}^{\varepsilon_{0}} \psi_{z_{0}, \varepsilon}(t) d t<\infty
$$

and

$$
\int_{\varepsilon<\left|z-z_{0}\right|<\varepsilon_{0}} K_{\mu}^{T}\left(z, z_{0}\right) \psi_{z_{0}, \varepsilon}^{2}\left(\left|z-z_{0}\right|\right) d x d y=o\left(I_{z_{0}}^{2}(\varepsilon)\right)
$$

as $\varepsilon \rightarrow 0$. Then the Beltrami equation (1) has a ring solution $f_{\mu}$.

Proof. Fix $z_{1}$ and $z_{2}$ in $D$. For $n \in \mathbb{N}$, define $\mu_{n}: D \rightarrow \mathbb{C}$ by letting $\mu_{n}(z)=$ $=\mu(z)$ if $|\mu(z)| \leq 1-1 / n$ and 0 otherwise. Let $f_{n}: D \rightarrow \mathbb{C}$ be a homeomorphic ACL solution of (1), with $\mu_{n}$ instead of $\mu$, which fixes $z_{1}$ and $z_{2}$. Such $f_{n}$ exists by the well-known existence theorem in the nondegenerate case, see e.g. [48, p. 98], cf. [7, p. 185 and 194]. By Proposition 1 and Lemma 2, in view of (32), the sequence $f_{n}$ is equicontinuous and hence by the Arzela-Ascoli theorem, see e.g. [49, p. 267], and [50, p. 382], it has a subsequence, denoted again by $f_{n}$, which converges locally uniformly to some nonconstant mapping $f$ in $D$. Then, by Theorem 3.1 and Corollary 5.12 in [27] on convergence, $f$ is $K(z)$-qc with $K(z)=K_{\mu}(z)$ and $f$ satisfies (1) a.e. Thus, $f$ is 
a homeomorphic ACL solution of (1). Moreover, by Propositions 1 and $2 f$ is a ring $Q$-homeomorphism, see (14), with $Q(z)=K_{\mu}^{T}\left(z, z_{0}\right)$ at every point $z_{0} \in D$.

Since the locally uniform convergence $f_{n} \rightarrow f$ of the sequence $f_{n}$ is equivalent to the continuous convergence, i.e., $f_{n}\left(z_{n}\right) \rightarrow f\left(z_{0}\right)$ if $z_{n} \rightarrow z_{0}$, see [49, p. 268], and since $f$ is injective, it follows that $g_{n}=f_{n}^{-1} \rightarrow f^{-1}=g$ continuously, and hence locally uniformly. By a change of variables which is permitted because $f_{n}$ and $g_{n}$ are in $W_{\text {loc }}^{1,2}$, see e.g. [7, p. 121, 128, 130, and 150], we obtain that for large $n$

$$
\int_{B}\left|\partial g_{n}\right|^{2} d u d v=\int_{g_{n}(B)} \frac{d x d y}{1-\left|\mu_{n}(z)\right|^{2}} \leq \int_{B^{*}} Q(z) d x d y<\infty
$$

where $B^{*}$ and $B$ are relatively compact domains in $D$ and in $f(D)$, respectively, such that $g(\bar{B}) \subset B^{*}$. The relation (33) implies that the sequence $g_{n}$ is bounded in $\mathrm{W}^{1,2}(B)$, and hence $f^{-1} \in \mathrm{W}_{\text {loc }}^{1,2}(f(D))$, see e.g. [51, p. 319].

Remark 2. If $f_{\mu}$ is as in Lemma 3 , then $f_{\mu}^{-1}$ is locally absolutely continuous and preserves nulls sets, and $f_{\mu}$ is regular a.e., i.e., differentiable with $J_{f_{\mu}}(z)>0$ a.e. Indeed, the assertion about $f_{\mu}^{-1}$ follows from the fact that $f_{\mu}^{-1} \in \mathrm{W}_{\text {loc }}^{1,2}$, see [7, p. 131 and 150]. As an ACL mapping $f_{\mu}$ has a.e. partial derivatives and hence by [6] it has a total differential a.e. Let $E$ denote the set of points of $D$ where $f_{\mu}$ is differentiable and $J_{f_{\mu}}(z)=0$, and suppose that $|E|>0$. Then $\left|f_{\mu}(E)\right|>0$, since $E=f_{\mu}^{-1}\left(f_{\mu}(E)\right)$ and $f_{\mu}^{-1}$ preserves null sets. Clearly $f_{\mu}^{-1}$ is not differentiable at any point of $f_{\mu}(E)$, contradicting the fact that $f_{\mu}^{-1}$ is differentiable a.e.

Corollary 3. Let $\mu: D \rightarrow \mathbb{C}$ be a measurable function with $|\mu(z)|<1$ a.e., $K_{\mu} \in$ $\in L_{\mathrm{loc}}^{1}$, and let $\psi:(0, \infty) \rightarrow(0, \infty)$ be a measurable function such that for all $0<t_{1}<$ $<t_{2}<\infty$

$$
0<\int_{t_{1}}^{t_{2}} \psi(t) d t<\infty, \quad \int_{0}^{t_{2}} \psi(t) d t=\infty .
$$

Suppose that for every $z_{0} \in D$ there is $\varepsilon_{0}<\operatorname{dist}\left(z_{0}, \partial D\right)$ such that

$$
\int_{\varepsilon<\left|z-z_{0}\right|<\varepsilon_{0}} K_{\mu}(z) \psi^{2}\left(\left|z-z_{0}\right|\right) d x d y \leq O\left(\int_{\varepsilon}^{\varepsilon_{0}} \psi(t) d t\right)
$$

as $\varepsilon \rightarrow 0$. Then (1) has a ring solution.

Lemmas 1 and 3 yield the following theorem by choosing

$$
\psi_{z_{0}, \varepsilon}(t)=\frac{1}{t \log \frac{1}{t}} .
$$

Theorem 4. Let $\mu: D \rightarrow \mathbb{C}$ be a measurable function with $|\mu(z)|<1$ a.e. and $K_{\mu} \in L_{\mathrm{loc}}^{1}$. Suppose that every point $z_{0} \in D$ has a neighborhood $U_{z_{0}}$ such that

$$
K_{\mu}^{T}\left(z, z_{0}\right) \leq Q_{z_{0}}(z) \quad \text { a.e. }
$$

for some function $Q_{z_{0}}(z)$ of finite mean oscillation at the point $z_{0}$ in the variable $z$. Then the Beltrami equation (1) has a ring solution.

The following theorem is an immediate consequence of Theorem 4. 
Theorem 5. Let $\mu: D \rightarrow \mathbb{C}$ be a measurable function with $|\mu(z)|<1$ a.e. and $K_{\mu} \in L_{\text {loc }}^{1}$. Suppose that at every point $z_{0} \in D$

$$
\varlimsup_{\varepsilon \rightarrow 0} f_{D\left(z_{0}, \varepsilon\right)} \frac{\left|1-\frac{\overline{z-z_{0}}}{z-z_{0}} \mu(z)\right|^{2}}{1-|\mu(z)|^{2}} d x d y<\infty .
$$

Then the Beltrami equation (1) has a ring solution $f_{\mu}$.

Applying Lemma 3 with $\psi(t)=1 / t$, we have also the following statement which is formulated in terms of the logarithmic mean, see (29), of $K_{\mu}^{T}\left(z, z_{0}\right)$ over the annuli $A(\varepsilon)=\left\{z \in \mathbb{C}: \varepsilon<\left|z-z_{0}\right|<\varepsilon_{0}\right\}$ for a fixed $\varepsilon_{0}=\delta\left(z_{0}\right) \leq \operatorname{dist}\left(z_{0}, \partial D\right)$.

Theorem 6. Let $\mu: D \rightarrow \mathbb{C}$ be a measurable function with $|\mu(z)|<1$ a.e. and $K_{\mu} \in L_{\mathrm{loc}}^{1}$. If at every point $z_{0} \in D$ the logarithmic mean of $K_{\mu}^{T}$ over $A(\varepsilon)$ does not converge to $\infty$ as $\varepsilon \rightarrow 0$, i.e.,

$$
\liminf _{\varepsilon \rightarrow 0} M_{\log }^{K_{\mu}^{T}}(\varepsilon)<\infty,
$$

then the Beltrami equation (1) has a ring solution.

Corollary 4. Let $\mu: D \rightarrow \mathbb{C}$ be a measurable function with $|\mu(z)|<1$ a.e. and $K_{\mu} \in L_{\text {loc }}^{1}$. Denote by $q_{z_{0}}^{T}(t)$ the mean value of $K_{\mu}^{T}\left(z, z_{0}\right)$ over the circle $C=\{z \in$ $\left.\in \mathbb{C}:\left|z-z_{0}\right|=t\right\}$. If

$$
\int_{0}^{\delta\left(z_{0}\right)} q_{z_{0}}^{T}(t) \frac{d t}{t}<\infty
$$

at every point $z_{0} \in D$ for some $\delta\left(z_{0}\right)>0$, then (1) has a ring solution.

Lehto considers in [2] degenerate Beltrami equations in the special case where the singular set $S_{\mu}$

$$
S_{\mu}=\left\{z \in \mathbb{C}: \lim _{\varepsilon \rightarrow 0}\left\|K_{\mu}\right\|_{L^{\infty}(D(z, \varepsilon))}=\infty\right\}
$$

of the complex coefficient $\mu$ in (1) is of measure zero, and shows that, if for every $z_{0} \in \mathbb{C}$ and every $r_{1}$ and $r_{2} \in(0, \infty)$ the integral

$$
\int_{r_{1}}^{r_{2}} \frac{d r}{r\left(1+q_{z_{0}}^{T}(r)\right)}, \quad r_{2}>r_{1},
$$

is positive and tends to $\infty$ as either $r_{1} \rightarrow 0$ or $r_{2} \rightarrow \infty$ where

$$
q_{z_{0}}^{T}(r)=\frac{1}{2 \pi} \int_{0}^{2 \pi} \frac{\left|1-e^{-2 i \vartheta} \mu\left(z_{0}+r e^{i \vartheta}\right)\right|^{2}}{1-\left|\mu\left(z_{0}+r e^{i \vartheta}\right)\right|^{2}} d \vartheta
$$

then there exists a homeomorphism $f: \overline{\mathbb{C}} \rightarrow \overline{\mathbb{C}}$ which is ACL in $\mathbb{C} \backslash S_{\mu}$ and satisfies (1) a.e. Note that the integrand here is the tangential dilatation $K_{\mu}^{T}\left(z, z_{0}\right)$, see (4).

We present now an extension of Lehto's existence theorem which enables to derive many other existence theorems as it was shown in [4]. In this extension we prove the existence of a ring solution in a domain $D \subset \mathbb{C}$ which by the definition is ACL in $D$ and not only in $D \backslash S_{\mu}$. Note that, in the following theorem, the situation where $S_{\mu}=D$ is possible. Note also that the condition (41) in the following theorem is weaker than the condition in Lehto's existence theorem. 
Theorem 7. Let $D$ be a domain in $\mathbb{C}$ and let $\mu: D \rightarrow \mathbb{C}$ be a measurable function with $|\mu(z)|<1$ a.e. and $K_{\mu} \in L_{\mathrm{loc}}^{1}$. Suppose that at every point $z_{0} \in D$

$$
\int_{0}^{\delta\left(z_{0}\right)} \frac{d r}{r q_{z_{0}}^{T}(r)}=\infty
$$

where $\varepsilon_{0}=\delta\left(z_{0}\right)<\operatorname{dist}\left(z_{0}, \partial D\right)$ and $q_{z_{0}}^{T}(r)$ is the mean of $K_{\mu}^{T}\left(z, z_{0}\right)$ over $\left|z-z_{0}\right|=r$. Then the Beltrami equation (1) has a ring solution.

Proof. Theorem 7 follows from Lemma 3 by special choosing the functional parameter

$$
\psi_{z_{0}, \varepsilon}(t) \equiv \psi_{z_{0}}(t):= \begin{cases}1 /\left[t q_{z_{0}}^{T}(t)\right], & t \in\left(0, \varepsilon_{0}\right), \\ 0, & \text { otherwise. }\end{cases}
$$

Corollary 5. If $K_{\mu} \in L_{\mathrm{loc}}^{1}$ and at every point $z_{0} \in D$

$$
q_{z_{0}}^{T}(r)=O\left(\log \frac{1}{r}\right) \quad \text { as } \quad r \rightarrow 0,
$$

then (1) has a ring solution.

Since $K_{\mu}^{T}\left(z, z_{0}\right) \leq K_{\mu}(z)$ we obtain, in particular, as a consequence of Theorem 7 the following result which is due to Miklyukov and Suvorov [38] for the case $K_{\mu} \in L_{\mathrm{loc}}^{p}$, $p>1$.

Corollary 6. If $K_{\mu} \in L_{\mathrm{loc}}^{p}$ for $p \geq 1$ and (41) holds for $K_{\mu}(z)$ instead of $K_{\mu}^{T}\left(z, z_{0}\right)$ for every point $z_{0} \in D$, then (1) has a $W_{\mathrm{loc}}^{1, s}$ homeomorphic solution with $s=2 p /(p+1)$.

Since the maximal dilatation dominates the tangential dilatation, all the above results obviously imply similar existence theorems in terms of conditions on the maximal dilatation established earlier in [30] which are important particular cases, see also the survey [39].

Remark 3. Note that all the distortion estimates for $Q$-homeomorphisms given in Section 2 can be applied to ring solutions from the above existence theorems. Indeed, in these theorems, for every domain $D \subset \mathbb{C}$ and every pairs of points $z_{1}$ and $z_{2} \in D$ and $w_{1}$ and $w_{2} \in \mathbb{C}$, there exist ring solutions with the normalization $f\left(z_{1}\right)=w_{1}$ and $f\left(z_{2}\right)=w_{2}$. The estimates hold for such solutions with $Q(z)=K_{\mu}^{T}\left(z, z_{0}\right)$ and $\Delta=$ $=\min \left(1 /\left(1+\left|w_{1}\right|^{2}\right)^{1 / 2}, 1 /\left(1+\left|w_{2}\right|^{2}\right)^{1 / 2}\right)$ and, in particular, if $w_{1}=0$ and $w_{2}=w_{0}$, then $\Delta=1 /\left(1+\left|w_{0}\right|^{2}\right)^{1 / 2}$. For ring solutions with $\left|f\left(z_{1}\right)\right|$ and $\left|f\left(z_{2}\right)\right| \leq R<\infty$, $\Delta=1 /\left(1+R^{2}\right)^{1 / 2}$.

1. Reich E., Walczak H. On the behavior of quasiconformal mappings at a point // Trans. Amer. Math. Soc. - 1965. - 117. - P. $338-351$.

2. Lehto O. Homeomorphisms with a prescribed dilatation // Lect. Notes Math. - 1968. - 118. - P. 58 - 73.

3. Gutlyanskii V., Martio O., Sugawa T., Vuorinen M. On the degenerate Beltrami equation // Trans. Amer. Math. Soc. - 2005. - 357. - P. 875-900.

4. Ryazanov V., Srebro U., Yakubov E. On ring solutions of Beltrami equation // J. Anal. Math. - 2005. -96. - P. $117-150$.

5. Maz’ya V. Sobolev classes. - Berlin; New York: Springer, 1985.

6. Gehring F. W., Lehto $O$. On the total differentiability of functions of a comlex variable // Ann. Acad. Sci. Fenn. Math. AI. - 1959. - 272. - P. 1 -9. 
7. Lehto O., Virtanen K. Quasiconformal mappings in the plane. - New York etc.: Springer, 1973.

8. Reimann H. M., Rychener T. Funktionen Beschrankter Mittlerer Oscillation. - Berlin etc.: Springer, 1975.

9. John F., Nirenberg L. On functions of bounded mean oscillation // Communs Pure and Appl. Math. 1961. - 14. - P. $415-426$.

10. Astala K. A remark on quasiconformal mappings and BMO-functions // Mich. Math. J. - 1983. - 30. P. 209-212.

11. Astala K., Gehring F. W. Injectivity, the BMO norm and the universal Teichmuller space // J. Anal. Math. - 1986. - 46. - P. $16-57$

12. Gehring F.W. Characteristic Properties of Quasidisk. - Montreal: Presses Univ. de Montreal, 1982.

13. Jones P. M. Extension theorems for BMO // Indiana Univ. Math. J. - 1980. - 29. - P. 41-66.

14. Martio O., Ryazanov V., Vuorinen M. BMO and injectivity of space quasiregular mappings // Math. Nachr. - 1999. - 205. - P. 149-161.

15. Reimann H. M. Functions of bounded mean oscillation and quasiconformal mappings // Comment. math. helv. - 1974. - 49. - P. 260-276.

16. Astala K., Iwaniec T., Koskela P., Martin G. Mappings of BMO-bounded distortion // Math. Ann. - 2000. -317. - P. $703-726$.

17. Iwaniec T., Koskela P., Martin G. Mappings of BMO-distortion and Beltrami type // J. Anal. Math. -2002. - 88. - P. $337-381$.

18. Iwaniec T., Martin G. Geometrical function theory and nonlinear analysis. - Oxford: Clarendon Press, 2001.

19. Sastry S. Boundary behavior of BMO-QC automorphisms // Isr. J. Math. - 2002. - 129. - P. 373-380.

20. Sarason D. Functions of vanishing mean oscillation // Trans. Amer. Math. Soc. - 1975 - 207. - P. 391 405.

21. Chiarenza F., Frasca M., Longo P. $W^{2, p}$-solvability of the Dirichlet problem for nondivergence elliptic equations with VMO coefficients // Ibid. - 1993. - 336, № 2. - P. 841 - 853.

22. Iwaniec T., Sbordone C. Riesz transforms and elliptic PDEs with VMO coefficients // J. Anal. Math. 1998. - 74. - P. $183-212$.

23. Palagachev D. K. Quasilinear elliptic equations with VMO coefficients // Trans. Amer. Math. Soc. -1995. - 347, № 7. - P. $2481-2493$.

24. Ragusa M. A. Elliptic boundary value problem in vanishing mean oscillation hypothesis // Comment. math. Univ. Carol. - 1999. - 40, № 4. - P. 651 - 663 .

25. Brezis H., Nirenberg L. Degree theory and BMO. I. Compact manifolds without boundaries // Selecta Math. (N. S.). - 1995. - 1, № 2. - P. 197-263.

26. Cianchi A., Pick L. Sobolev embeddings into BMO, VMO, and $L_{\infty} / /$ Ark. mat. - 1998. - 36, № 2. P. $317-340$.

27. Ryazanov V., Srebro U., Yakubov E. BMO-quasiconformal mappings // J. Anal. Math. - 2001. - 83. P. $1-20$.

28. Ryazanov V., Srebro U., Yakubov E. Plane mappings with dilatation dominated by functions of bounded mean oscillation // Sib. Adv. Math. - 2001. - 11, № 2. - P. 94-130.

29. Ignat'ev A., Ryazanov V. Finite mean oscillation in the mapping theory // Ukr. Math. Bull. - 2005. - 2, № 3. - P. $403-424$.

30. Ryazanov V., Srebro U., Yakubov E. Finite mean oscillation and the Beltrami equation // Isr. J. Math. 2006. - 153. - P. $247-266$.

31. Ryazanov V., Srebro U., Yakubov E. The Beltrami equation and FMO functions // Contemp. Math. - 2005. - 382. - P. 247-266.

32. David G. Solutions de l'equation de Beltrami avec $|\mu|_{\infty}=1 / /$ Ann. Acad. Sci. Fenn. Ser. AI. Math. AI. - 1988. - 13, № 1. - P. $25-70$.

33. Tukia P. Compactness properties of $\mu$-homeomorphisms // Ibid. -1991 . - 16, № 1. - P. 47-69.

34. Brakalova M. A., Jenkins J. A. On solutions of the Beltrami equation // J. Anal. Math. - 1998. - 76. P. $67-92$.

35. Kruglikov V. I. The existence and uniqueness of mappings that are quasiconformal in the mean // Metric Questions of the Theory of Functions and Mappings. - Kiev: Naukova Dumka, 1973. - P. 123 - 147.

36. Martio O., Miklyukov V. On existence and uniqueness of the degenerate Beltrami equation // Complex Variables Theory Appl. - 2004. - 49. - P. 647-656. 
37. Miklyukov V. M., Suvorov G. D. On existence and uniqueness of quasiconformal mappings with unbounded characteristics // Investigations in the Theory of Functions of Complex Variables and its Applications. Kiev: Inst. Math. Acad. Sci. USSR, 1972.

38. Pesin I. N. Mappings quasiconformal in the mean // Dokl. Akad. Nauk SSSR. - 1969. - 187, № 4. P. $740-742$.

39. Srebro U., Yakubov E. The Beltrami equation // Complex Analysis, Geometry, Function Theory. - Amsterdam: Elsevier, 2005. - Vol. 2. - P. 555-597.

40. Saks S. Theory of the integral. - New York: Dover Publ. Inc., 1964.

41. Martio O., Ryazanov V., Srebro U., Yakubov E. Q-homeomorphisms // Contemp. Math. - 2004. - 364. P. $193-203$.

42. Martio O., Ryazanov V., Srebro U., Yakubov E. Mappings with finite length distortion // J. Anal. Math. 2004. - 93. - P. 215-236.

43. Martio O., Ryazanov V., Srebro U., Yakubov E. On Q-homeomorphisms // Ann. Acad. Sci. Fenn. - 2005. - 30. - P. 49-69.

44. Gehring F. W. A remark on the moduli of rings // Comment. math. helv. - 1961. - 36. - P. $42-46$.

45. Vaisala $J$. Lectures on $n$-dimensional quasiconformal mappings // Lect. Notes Math. - Berlin etc.: Springer, 1971. - 229.

46. Gehring F. W. Rings and quasiconformal mappings in space // Trans. Amer. Math. Soc. - 1962. - 103. P. $353-393$.

47. Heinonen J., Koskela P. Sobolev mappings with integrable dilatations // Arch. Ration. Mech. and Anal. 1993. - 125. - P. $81-97$.

48. Ahlfors L. V. Lectures on quasiconformal mappings. - Princeton etc.: D. Van Nostrand Company, Inc., 1966.

49. Dugundji J. Topology. - Boston: Allyn and Bacon, Inc., 1966.

50. Dunford N., Schwartz J. T. Linear operators. Pt I: General theory. - New York; London: Int. Publ., Inc., 1957.

51. Reshetnyak Yu. G. Space mappings with bounded distortion // Transl. Math. Monographs, AMS. - 1989. -73 .

Received 29.06.2006 